\title{
El agua en la dinámica de los recursos naturales estratégicos en un mundo volátil, incierto, complejo y ambiguo
}

\author{
JUAN ERARDo BATTALEME*
}

\begin{abstract}
RESUMEN
La idea del agua como recurso natural estratégico de la política internacional no es nueva, sin embargo, desde la primera década del corriente siglo el acceso a la misma ha cobrado especial intensidad. Por ello, el presente artículo pone en valor la idea del agua como recurso mediante el uso del concepto de hidropolítica, que separa al mito de las llamadas guerras por el agua de la realidad geopolítica que lo trasunta. Al enfatizar en la dinámica conflictiva, y en especial en la aspiración de las grandes potencias para hacerse del recurso, queda marginada la discusión más apremiante -al entender del autorque es identificar cómo el recurso afecta a los procesos de la política regional y global de manera diferenciada a partir de ponderaciones completamente distintas entre los actores que tienen algún interés en este recurso. Siguiendo las premisas del realismo, existe una mayor probabilidad de que se produzcan conflictos en torno al agua a partir de su uso compartido que por la intervención directa de un actor extra regional para tomar el control de la misma. En efecto, este artículo tiene por objetivo discutir cuándo un recurso natural estratégico es considerado como un factor de conflicto armado, ya sea inter o intra estatal, para luego trasladar ese debate a la hidropolítica y comprobar si su importancia relativa hace o no que la dinámica que gira en torno a ella sea diferente a la existente alrededor de otros recursos.
\end{abstract}

En este sentido, esperamos demostrar al menos en las discusiones existentes y con la evidencia que muestra el relevamiento sobre lo reflexionado que, en relación al agua, la potencialidad existente por los juegos de suma cero parece ceder su lugar a construir regímenes internacionales que administren o encapsulen al conflicto. Si en un periodo determinado aparecen lecturas o percepciones que hacen temer por un futuro donde la escasez provoque conflictos, el cambio tecnológico y la arquitectura institucional permiten contemplar un espacio para la resolución de las divergencias que puedan surgir sin que la sangre llegue al río.

\section{Palabras clave}

Recursos naturales estratégicos; geopolítica; hidropolítica; conflictos internacionales; teoría realista.

\section{TitLE}

Water in the dynamics of strategic natural resources in a volatile, uncertain, complex and ambiguous world

\begin{abstract}
Are we close to facing what some researchers and politicians call a "war for water as a resource"? The ideas around this topic are divided.We think that external factors, such as climate change and the unequal distribution of the resource, will create conditions and pressures that shape perceptions and will eventually affect political behavior that will lead to war. Others agree that there are changes in the political landscape. However, when we look at different resources, we must be aware that they present different dynamics, affecting in different ways the political behavior of actors related to specific resources. War is not necessarily a consequence of resource necessity and access problems; resource wars are related to external factors like opportunity and power asymmetries, among others.
\end{abstract}

In the case of water, if we consider the scarcity problem, technological change and the capacity to create institutional ways to regulate its use, we can avoid the worst consequences of the scramble for resources.And even if access and necessity

\section{DOI:}

https://doi.org//0.15366/relacionesinternacionales2020.45.004

Formato de citación recomendado:

BATTALEME, Juan Erardo (2020). "El agua en la dinámica de los recursos naturales estratégicos en un mundo volátil, incierto, complejo y ambiguo”, Relaciones Internacionales, n 45 , pp. 91 - 105.
* Juan Erardo BATTALEME, Magíster en Relaciones Internacionales por la FLACSO

(Argentina) y

Magister en

Ciencias del Estado por la UCEMA (Argentina). Secretario

Académico del Consejo Argentino para las Relaciones Internacionales (CARI), Director de la Maestría en Defensa Nacional de la Facultad de la Defensa Nacional (FADENA). Profesor adjunto y titular en varias universidades de grado y posgrado argentinas.

Especialista en política exterior, defensa $y$ ciberseguridad. Contacto: jbattaleme@ hotmail.com.

Recibido:

21/03/2020

Aceptado:

23/07/2020 
could be the causes of conflict, we will also find its roots in another parts of the dynamics of rivalries, as the conflict for the Golan Heights shows.

The present work tries to put into perspective the preceding discussion to clarify the current dynamics around different types of natural resources. To do that, we must start discussing what "Strategic Natural Resources (SNR)" are, what their role in geopolitical terms is, and to understand how they affect the political calculus around them. Resource discussions about policies, conflicts, and politics use mainly a realist approach, because it is all about use, availability, and scarcity. Security is an essential topic around these issues, but in the case of water, perspectives can be a little bit different.

We should consider Hydropolitics as a topic within the geopolitical field of studies. In that sense, it shows how different the dynamics around water are, and even if many conflicts of interest surround it, most of the time political action is not to fight over it in the sense of going to war.We will see tensions but no war.A war over water is considered awful for everyone, so it is more useful to try to channel the conflict to a more productive arena. In that sense, the creation of regimes, norms, and treaties around a shared river, underground water deposit, sustainable use, and others will be the most critical aspects of policy coordination around those who have a dispute over it. Moreover, we will see others trying to assist major partners in managing the problems that its uses generate. Policymakers look at social and economic considerations surrounding war over water, which acts as a political barrier most of the time, meaning they are able to prevent it.

The objectives of the present article are twofold, and they are interrelated. The first one is to advance the discussion around natural resources and how they become a problem that could lead to war. Geopolitics and Realist Theory are the best tools to obtain a comprehensive picture of how these resources become factors in war.We can thus tackle the issue of war for water, which is different from a conflict over water as such. When we look at security considerations, realism believes that resources are a contributing factor to the balance of power, but also that they do not affect the security problematic. When we look at the accumulative power of water as a resource, we will find it to be "neutral" in the sense that does not affect the fragile balance of power among potential rivals.

The second objective of the article is different, but related. Water is a slightly different kind of SNR. Not only does it create conflict, but it also creates rivalries among those who perceive that they are going to face access problems to the resource. At the same time, however, it does not push to resolve the situation violently, through for instance interstate conflict. Conflict around the resource is a little bit more intense when we look at specific countries, but the differences in how this are solved stem from state capacity. If in an interstate conflict its accumulation is neutral, in an internal conflict the accumulation power could be positive or negative, affecting the internal balance and creating more space for internal war.

As a methodology, we use a descriptive and qualitative analysis of previously published work with the recent data around the issue to create a lucid and differentiated view when we discuss war over water resources. Water is not the best example to support views that emphasize a world prone to war. Moreover, we can say that there are fewer incentives to go to war for water than other resources. But the reader should be aware that the kind of insights we extract here do not lead to a liberal approach to water governance.A realist approach to finite cooperation is more useful to maintain stability. Water is strategic and related to territoriality, so identity considerations apply to hydropolitics, meaning that states are important stakeholders in this issue.

Nevertheless, we could think that some tension would arise between neighbors who share water resources. It is much more difficult, however, for a great power to take steps towards war over water. If they need it, they can pay for access to it. Even so, internally, the states could face conflicts around the resource. This difference is significant because we spend too much time talking and thinking about the international dimension of the problem when we should look at the internal dimension of it.With this in mind, the international discussion around water will be different and possibly better. Consider, for example, water contamination as a result of corporate activities. Neighbor states create a regime to overlook how, when, and to what degree they pollute the shared resource. They establish limits around the river and coastal activities, they enforce it, and they get around the table to deal with previous unconsidered situations. Furthermore, when differences arise, they stick to their commitments, even when the military balance is in favor of one side.

We will divide the following article into three short and related discussions. The first part puts into perspective the discussion around conflict and resources, giving water the specific place that it deserves. The second part revolves around the geopolitics of location, whereby availability and scarcity are at the center of the discussion.Within this section, we will take a hydropolitics perspective to talk about the use of water.This is important because we will end our discussion looking at the differences between this resource and others, for example energy.

With that in mind, this article serves as an introductory reading around how we think, act, and develop policy related to natural resources, showing that war is not the only possible result of resource competition.

\section{KEYWORDS}

Strategic natural resources; geopolitics; hydropolitics; international conflicts; realist theory. 
"Michael Burry, se contactó varias veces con diversas agencias del gobierno para explicarles cómo supo del colapso de los mercados años antes de que efectivamente sucedieran. Las inversiones que aún realiza se concentran en un solo recurso: agua"

(McKay, 20I5).

Al final de la película ganadora de un Oscar La gran apuesta, se menciona que Michael Burry solo se dedica a realizar operaciones bursátiles con el agua, remarcando la importancia que ésta tiene en la realidad económica, y por qué no militar, en el presente siglo. En este sentido y como podemos apreciar gracias al mapa cronológico de conflictos del agua del Worldwater.org, en 2019 se registraron en los últimos tres años ciento ochenta y ocho conflictos que involucraron recursos acuíferos y una duplicación de casos si comparamos la década 2010-2019 (cuatrocientos sesenta y cinco casos en total) versus 2000-2009 (doscientos veintidós casos en total).

Como recurso siempre ha sido un elemento vital en las actividades humanas, pero por múltiples razones ha quedado desdibujada en las consideraciones generales cuando se habla de recursos naturales junto con geopolítica. No obstante, en épocas recientes ha comenzado a considerársela estratégica.

Por ello, el presente artículo tiene por objeto discutir qué consideramos Recursos Naturales Estratégicos (en adelante, RNE), enfocándonos en el agua, para entender que tal vez es uno de los pocos que retiene una centralidad para todos los integrantes del juego económico, político y militar en el sistema internacional; aunque en este caso particular su condición estratégica varía según el uso que tenga en su proyección política internacional.

Frente a un escenario internacional multipolar, con las potencias emergentes incidiendo en el mercado internacional de materias primas, con Estados Unidos señalando que no depende del petróleo de Oriente para asegurar su posición energética, y con una discusión sobre el acceso a mercados y recursos, se reaviva la discusión política y teórica sobre la relación causal que tienen los recursos naturales en la conflictividad internacional. Esto se debe a la propensión de las grandes potencias hacia su control y explotación dado el carácter posicional que los mismos poseen. En definitiva, los recursos son una expresión material de las fortalezas y de las vulnerabilidades que poseen los estados en el escenario internacional.

De este modo, los RNE y el acceso, por ejemplo, a fuentes de agua potable, son cartas de poder que otorgan ventajas posicionales, transformándose en fuentes transaccionales centrales dependiendo de las necesidades económicas y políticas de los estados que se ven involucrados en la dinámica de los intercambios. Esta situación, se traduce en las reasignaciones de las zonas de influencia y de control sobre las cuales las potencias buscan asentarse valiéndose de aquellos recursos que consideran estratégicos al comprender que obtendrán por dicho posicionamiento una ventaja mayor en las condiciones internacionales emergentes. Asimismo, cuando hablamos de recursos, es más frecuente pensar en juegos de suma cero que en términos positivos, a menos que se trate del problema de aversión común como suele suceder con los diversos bancos de pesca para evitar que sean depredados. 
Dos objetivos guían el presente trabajo: el primero, se centra en analizar la discusión geopolítica de los recursos estratégicos a los efectos de entender la lógica de su competencia actual en un contexto económico interdependiente. Si históricamente el resultado directo de las competencias interimperiales era por las zonas donde los recursos se asentaban. De esta manera se facilitaron una serie de conflictos armados de las potencias en las zonas periféricas y las políticas de conquistas territoriales. Actualmente la interdependencia productiva conocida como formación de cadenas de valor junto con la comercial ubica la competencia bajo la égida de la transnacionalización económica llevando a que sea más beneficioso el comercio que la conquista (Rosencrance, 1986) y el acceso antes que la posesión (Klare, 2003). El segundo objetivo busca responder acerca de las circunstancias según las cuales aquella competencia internacional puede degenerar en un conflicto armado, ya sea intra o inter estatal, en especial por el agua. La competencia por los polos, como se la conoce actualmente, en especial por las reservas de agua dulce en el Ártico, muestra que situaciones de escasez, sumadas a cambios relativos de poder suelen activar los conflictos que previamente se encontraban encapsulados, como se ha visto a diversas escalas en distintos lugares del planeta (Klare, 20I2). Esta situación y en especial las dinámicas relacionadas con el agua llevan a pensar cual puede ser el rol de las fuerzas armadas.

El escenario internacional presenta distintas características, entre ellas, una creciente perspectiva de escasez (Conca, 2005; Klare, 2012; Schweller, 1999), y una matriz tecnológica energética vinculada principalmente al consumo de combustibles fósiles'. Además, cuenta con una población mundial en crecimiento $y$, en consecuencia, debe enfrentar la necesidad de suplir a estos polos de dos grandes grupos de materias primas: en primer lugar, de alimentos (el cual incluye a los recursos hídricos) en un mundo donde producirlos cuesta cada vez más en términos de huella ambiental; en segundo lugar, de insumos para las industrias de diverso tipo aplicado a los múltiples usos que demandan minerales. Asimismo, es definitorio en la composición de dicho escenario internacional, la irrupción de un mundo multipolar donde sus competidores, a diferencia de etapas previas, están representados por todas las regiones del globo de modo que se constituyen nuevos centros y se construyen nuevas periferias (o, en otros términos: viejas periferias relacionadas con nuevos centros). Igualmente, a lo antedicho debe sumarse el problema del exceso de ruido político entre las sociedades, las discusiones vacuas pero fuertes entre los diversos sectores políticos y de la sociedad civil, y la posibilidad de transformarse mediante la vinculación de determinadas cuestiones (problemas de desarrollo, climáticos, y comerciales) en verdaderas pesadillas políticas para los decisores.

La dinámica vinculada a los auges y a las caídas de las potencias difícilmente cambie, al igual que su relación con los nuevos emergentes industriales, aun cuando hoy hablemos de la cuarta revolución industrial. Una nueva distribución de poder conlleva un reacomodamiento de los centros de poder que los obliga a asegurarse espacios que los provea de materias primas de manera sostenida, conformando periferias cercanas que se relacionan con su proyección de poder, y brindando a su vez, la protección adecuada a las líneas de comunicación centro periferia.

Así, las potencias emergentes y las establecidas presionan en espacios geográficos similares

La demanda mundial de energía primaria se eleva a cerca de 13.972 toneladas equivalentes de petróleo (por sus siglas en inglés, Mtoe), que se reparten de la siguiente manera: $32 \%$ para el petróleo, $27 \%$ para el carbón, $22 \%$ para el gas natural, el I $2 \%$ para energías renovables y el $5 \%$ para la energía nuclear. 
a los efectos de hacerse con los recursos que consideren necesarios para su crecimiento y para mantener los diferenciales de poder en el sistema internacional. De ahí que las periferias (Medio Oriente, Asia Central, África, América Latina) presenten la misma dinámica de competencia, sólo que, en la actualidad existen nuevos jugadores operando en esos espacios. Así, para responder entonces a qué entendemos por recursos naturales estratégicos debemos abordar tres preguntas centrales: ¿qué condiciones llevan a considerar determinadas materias primas como estratégicas? ¿Cuáles son los problemas derivados de su ubicación?Y en correspondencia, ¿cómo inciden en su relevancia estratégica?

El hecho de que transitemos un mundo que se referencia como volátil, incierto, complejo y ambiguo (VICA) impacta en cómo se trata la cuestión de los recursos naturales. En esencia, determinados recursos en base a su valor (real y perceptual) contribuyen a la volatilidad existente. Esto puede ejemplificarse en la subida del precio del petróleo ante un conflicto armado, lo cual, pone en acción un juego existente sobre la evolución de su posición a futuro, impactando en diversas agendas.Así, la complejidad tiene que ver con la intersección existente entre los mismos y dichas agendas. Otro ejemplo, es la producción de alimentos, que genera conflictos de distinta intensidad sobre los cursos de agua y el destino de los caudales necesarios para poder satisfacer diversas demandas preexistentes en la sociedad. Esto es incierto dado que no sabemos cuál es la sucesión de eventos que prosiguen una vez que una situación conflictual se pone en escena. Si antes pensábamos en términos lineares, hoy la no linealidad demanda un mayor ejercicio analítico.

En pocas palabras, la ambigüedad requiere de conductas prudentes, pero al mismo tiempo, de las suspicacias que demanda entender un mundo de intereses cruzados donde los recursos son compartidos mediante los complejos entramados societales que caracterizan a las relaciones transnacionales hoy, y que afectan tanto a dinámicas privadas como públicas.

Si miramos las razones por las cuales en general la competencia por RNE en general y en particular el agua dulce, presentan una dinámica que suele terminar en un conflicto armado, podemos invocar que los denominadores comunes son: falta de acuerdo en materia de estatus legal, sobre todo cuando hay un recurso compartido, crecimientos poblacionales, destrucción de ecosistemas, y más recientemente los problemas de afectación desigual del cambio climático.

Asimismo, se suma la llamada maldición por abundancia, que en el caso de los cursos de agua o acuíferos se traduce en el impacto desproporcionado que tienen ciertas cuencas compartidas sobre la proporción de recursos que un espacio tiene sobre otros y la manipulación que se puede hacer de los mismos, como sucede actualmente en el río Paraná y la bajante creciente de su caudal de agua, producto del control del curso que tiene Brasil en las etapas superiores del río.

\section{Recursos naturales estratégicos: una aproximación desde la teoría al problema del agua como RNE}

Cada país, dependiendo de su ubicación geográfica, su vecindad, sus necesidades y sus posibilidades considerará un determinado recurso como estratégico. Esto no significa desmerecer la preponderancia que tienen unos recursos sobre otros debido al impacto que éstos tienen en el sistema internacional. Sin embargo, en una dimensión local algunos de ellos pueden ser centrales 
para la convivencia o la supervivencia en dicho espacio, aun cuando su impacto internacional directo sea reducido.

Cuando pensamos en recursos estratégicos debemos hacerlo en función de elementos tan estrechamente relacionados que conforman un entramado compuesto por los actores involucrados, por los intereses de dichos actores (siendo éstos los que le dan un sentido según sus necesidades), y a su vez por los objetivos de, no sólo de los países que los representan, sino también, de la propia agenda de las empresas con intereses económicos sobre los recursos implicados.

El interrogante acerca de cuáles son los recursos que entran en esta categoría nos permite entender por qué algunos son considerados estratégicos, mientras que otros no. Responder a dónde están estos recursos nos va a llevar al correlato geográfico de las líneas de presión en las que estos son disputados, controlados y explotados, como así también a percibir aquellas futuras plausibles líneas de posicionamiento de los estados con intereses vinculados a ellos.Ambas preguntas obligan a poner al analista en el mapa a los efectos de poder desarrollar consideraciones geopolíticas derivadas, entendiendo dichas consideraciones como elección y no como mandato.

De esta manera, las consideraciones acerca de la relevancia de un recurso nos permiten ver que allí, donde esté ubicado, existirá una competencia, sea o bien por mantener dicho recurso disponible, o por la intención de obtener los beneficios de su control y explotación. Además, si bien la correlación entre recursos naturales y guerras esta históricamente bien datada, esa misma se ha debilitado en la actualidad, ya que los beneficios de una guerra para la obtención de recursos deben sobrepasar ampliamente los costos de su obtención.

Aún hoy existe la posibilidad de una guerra con objetivos limitados a los efectos de capturar recursos naturales de un país, aunque las probabilidades se hayan reducido entre grandes potencias tal como señala Stephen Brooks (1997), como consecuencia de sus capacidades militares. Sin embargo, entre actores débiles entre sí o poderosos en su relación con aquellos de menor peso, la existencia de recursos naturales, puede originar un conflicto armado, tal como lo explica Bremer (citado por Vázquez, 2000, p. 26) cuando hace un relato acerca de qué sabemos (y qué desconocemos) en relación a la emergencia de los conflictos armados. Al respecto, podemos identificar tres causas por las cuales los estados van a la guerra: elección, destino o coincidencia. En un mundo donde existen múltiples motivaciones y donde los recursos naturales son uno de ellos, este elemento ha perdido fuerza como causa de guerra entre estados. Pese a ello, la incidencia de los recursos naturales en los conflictos intertribales o étnicos es más consistente, y la posibilidad y la probabilidad de conflictos detonados por el uso, explotación, y control entre grupos domésticos, es un factor tenido en cuenta con creciente empeño por los grandes poderes.

\section{Los RNE: consideraciones acerca de su relevancia en la política doméstica e internacional}

El objetivo del presente apartado no es la enumeración específica de todos los recursos considerados estratégicos. Por el contrario busca trazar algunas consideraciones que permiten establecer cuándo algunos de estos pueden ser considerados estratégicos. La respuesta se 
relaciona con la lógica posicional que tienen los estados en el sistema internacional. En este sentido, Hans Morghentau, en su obra seminal Política entre las Naciones (1986) da cuenta de que la posición estructural que un país depende de determinados recursos materiales de poder, entre ellos la posesión de materias primas. La característica central del mundo de los recursos naturales es entonces su distribución desigual, tanto en la tenencia, como en el control de los mismos (Russett y Starr, 1992). En efecto, esa repartición se ve caracterizada por una concentración de determinados recursos en ubicaciones geográficas específicas.

La clasificación de recursos naturales estratégicos es una condición externa al recurso per se, estando directamente relacionada al grado de evolución tecnológica y a sus requerimientos en el proceso de utilización productivo junto con las necesidades básicas de supervivencia de los seres humanos debido a nuestras condiciones biológicas. En este sentido, la condición de abundancia o escasez es central en la formación de las percepciones para entender, no sólo la competencia por los recursos existentes, sino también su influencia en la forma en la que dicha competencia puede tener lugar.

Al respecto, se puede decir que un RNE no siempre es escaso, por ello, la abundancia no le resta importancia a un recurso, y por lo tanto, puede ser igualmente considerado estratégico. Lo interesante aquí es que dicha abundancia hace que el mismo no genere presiones competitivas masivas, ya que su provisión se encuentra relativamente asegurada. Por otra parte, los cambios tecnológicos pueden provocar que un recurso que previamente ha sido considerado estratégico pierda esta conceptualización y otro surja en substitución suya. Además, la escasez incorpora presiones competitivas que pueden degenerar en conflictos armados. Por este motivo, responder cuáles, demanda cruzar en el análisis: distribución, necesidad, posición estructural del demandante y concentración.

Si bien los RNE se modifican, es una constante histórica la búsqueda de aquellos que permitan desarrollar poder y garantizar la supervivencia del estado. Si los recursos naturales conforman parte de la base material de poder de los estados, la consecuencia lógica es la búsqueda, y si tradicionalmente se trataba de acapararlos, la preocupación actual es mantener el acceso -y poder denegárselo a otro de ser necesario-.

En correlación, con las materias primas se puede aspirar a buscar otros recursos que se necesiten y utilizarlos para su propio desarrollo. Esa es otra característica central para entender este apartado, ya que al pensar en cuáles son estratégicos tenemos que pensar también en la transferencia que los mismos permiten: de su uso, comercialización, explotación, etcétera, se obtiene lo necesario para adquirir recursos materiales no naturales, pero igual de estratégicos (por ejemplo: tecnología para desarrollar complejos industriales y/o armas).

Stephen Van Evera (1999, Pp. II0-II2), siguiendo este planteamiento, defiende que los recursos son estratégicos en la medida en que permiten obtener activos en otras áreas que contribuyen a la seguridad y al bienestar del estado, por lo tanto, la consideración esencial es su grado de acumulatividad en relación a otros recursos existentes. Si con el recurso, en este caso natural, obtengo otro elemento de poder material, el primero resulta estratégico para mi política y por lo tanto es un centro de preocupación a proteger, o llegado el caso a adquirir, ya que sin 
aquel bien uno pierde capacidad para actuar².

Los llamados juegos de suma cero suelen aplicarse sobre aquellos recursos que son considerados estratégicos. Esa situación es el resultado de la dinámica que existe entre quien los controla y quienes pugnan por controlarlos, las tensiones derivadas frente a un eventual agotamiento.

Una definición común sobre RNE suele ser sobre aquellos que son escasos o vitales para el desarrollo de la actividad económica y el mantenimiento del nivel de vida de un país. Aun cuando dicha explicación es útil, ya que podemos listar rápidamente una serie de recursos naturales, la misma resulta a su vez incompleta. Para poder completarla deberíamos sumarle las consideraciones sobre la distribución desigual, el grado de demanda y las posibilidades tecnológicas de reemplazo de dicho recurso. Por ejemplo, cuando hablamos de tierras raras las mismas presentan dos condiciones: son por un lado abundantes, pero como contrapartida, están ubicadas en un número relativamente bajo de países y quienes le dan mayor empleo son quienes no las poseen, obligando a tener que hacer acopio de dicho material. Otro escenario interesante es la relación entre la tierra arable para la producción de alimentos y la distribución de población, lo cual permite saber las necesidades de determinadas regiones y países de importar alimentos, siendo vulnerables a las disrupciones en la provisión de este recurso vital. Por último, se estima además que ciertos minerales - aquellos centrales para la industria- son escasos y están concentrados, lo cual supone altas presiones competitivas en el sistema internacional (Kouby et al., 2013).

Entonces, al considerar la capacidad de reemplazo, ya sea por existencias que se agotan o debido al cambio tecnológico (tanto en el uso de nuevos materiales cumpliendo función de reemplazo o en términos de eficiencia), nos obliga a considerar la utilización del mismo en función de los procesos productivos mejorados. La perspectiva de obtener energía de fuentes alternativas al petróleo representa una de las carreras tecnológicas más importantes, la cual tiene lugar de forma simultánea con la búsqueda de nuevas fuentes de petróleo que explotar. De esta forma, vemos como la explotación y el reemplazo coexisten, previendo o tratando de evitar las peores previsiones acerca del agotamiento del recurso.

Asimismo, cuando discutimos acerca de la preeminencia de un determinado recurso resulta importante ver quienes se constituyen entre los demandantes de los mismos. La construcción actual de hipótesis de conflictos que se relacionan con los recursos naturales combina la demanda real con la perspectiva futura de demanda. Esto se debe a: la incapacidad para reemplazar dichos recursos, a la incorporación de nuevos consumidores, y principalmente al acceso y a la disponibilidad de estos frente a los cambios en el entorno político y/o ambiental del mismo. Por tanto, las consideraciones acerca de lo estratégico resultan una suma de todos estos factores que confluyen sobre determinados recursos.

De este modo, para poder unir la cuestión de los RNE y la potencialidad para la conflictividad, resulta necesario fraccionar dicha demanda y su correlación con el conflicto en un

\footnotetext{
Aparte de la acumulabilidad positiva existen dos clasificaciones más: la acumulabilidad negativa, es un recurso que al tenerlo genera costos y no permite para el estado una transferencia positiva (por ejemplo, territorio contaminado); $y$ la acumulabilidad neutra, que no suma ni resta recursos al estado.Véase Van Evera (1999).
} 
aspecto global y local o regional. No todos los que demandan inciden de la misma manera en el sistema internacional, ni tienen las mismas capacidades para hacer una vinculación de cuestiones. Dicho de forma sencilla: una demanda insatisfecha por algún recurso que considera estratégico EEUU puede desencadenar una reacción muy distinta que aquella emanada por un país como Bolivia frente a la misma situación.

A partir de lo expuesto podemos deducir: en primer lugar, que en un contexto de economía transnacional el comercio de recursos prima sobre su extracción en base a la conquista, y que el mercado es una fuerza que opera sobre las consideraciones acerca de cuan estratégico resulta ser un determinado recurso en un momento dado. Esta es una diferencia fundamental entre las etapas previas del siglo XIX y el siglo XX; la obtención y el control de los recursos se da fundamentalmente en su relación privado y estado o bajo la conformación de consorcios híbridos estatales y privados. Hoy, los flujos de Inversión Extranjera Directa (IED) son una forma más efectiva que la conquista para sumar recursos (Poelhekke y Van Der Ploeg, 20I0). En segundo lugar, y desde el plano de la seguridad, si consideramos que los recursos naturales tienen una disposición geográfica fija y que sobre ellos existe una competencia, la conflictividad va a estar mediada por la premisa acerca de donde están ubicados, quién tiene la posesión física y quién los necesita.

El poseedor de recursos puede enfrentar dos variantes de amenazas: una regional o local, y otra extrarregional o global. La primera implica que aquel que demanda un determinado recurso sea un vecino y que, además, se encuentre en una situación de poder tal que le permita obtenerlo si aparecen obstrucciones a su acceso; por lo general puede provenir de una potencia regional que se encuentra en un proceso de creación de periferia cercana, o bien localmente, cuando la ubicación del recurso permite a grupos lanzar desafíos activos contra el gobierno acelerando procesos de descomposición política del estado en cuestión. Ejemplo de ello es la creciente disputa por los recursos hídricos que se dan entre Argentina y Brasil por el río Paraná y la bajante que se produce por los acopíos que realiza ese país sobre el curso superior del río. Otro ejemplo son las constantes ejercitaciones militares que Brasil realiza, a veces solo, a veces con Paraguay sobre Itaipú.

La segunda variante, como ya se ha dicho, puede provenir de un ámbito extrarregional - global. Según Buzan (2004, pp. 69-7I) puede ser de una superpotencia o de un gran poder. Estos actores poseen las capacidades militares necesarias para apropiarse de los recursos, lo que sumado a la incapacidad por parte del poseedor de los mismos para defenderlos de manera adecuada y de disuadir a estos actores de operar en sus espacios soberanos, constituye una amenaza para el país que detenta el recurso estratégico. La extracción creciente de agua dulce (vía témpanos), por parte de Rusia es un buen ejemplo de cómo los grandes poderes utilizan su posición relativa de poder para actuar en detrimento de actores menores con reclamos sobre un determinado recurso.

Esta diferenciación resulta importante por dos consideraciones políticas en relación a los recursos naturales. La primera tiene que ver con la posibilidad de generar una agenda positiva en relación a su uso, debido a que, si se percibe que las presiones por la obtención de un determinado recurso no provienen de la vecindad inmediata, existe una mayor factibilidad de 
preparar argumentaciones que reafirmen la integración y la defensa común de estos. En segundo término, se pone de manifiesto quién o quiénes pretenden determinados recursos permitiendo visualizar la amenaza y las posibles respuestas en términos de su defensa.

En consecuencia, desde la perspectiva de quien los necesita, su seguridad se encuentra amenazada, primeramente, por la cercanía o lejanía de sus fuentes de provisión de recursos naturales donde problemas como el acceso, la negación de rutas comerciales o las amenazas a su transporte, se vuelven parte de sus consideraciones al momento de optar por las zonas donde van a concentrar sus esfuerzos (inclusive militares) en la obtención de recursos. Segundo, la voluntad de proveer dicho recurso por parte del productor se presenta como una plausible amenaza desde este punto de vista, siendo que tanto los cambios políticos, la violencia en determinadas áreas, y todo lo que lleve a la disrupción en la provisión normal por parte del productor afecta las relaciones entre el productor y el consumidor.

Entonces, al poder interrelacionar los recursos con la relación existente entre demanda, oferta y acceso, podemos escapar de los enfoques extremos que priman en relación a los recursos naturales. La fuerza argumentativa presenta una versión en exceso militarista, donde las tensiones por la creciente escasez llevarán inevitablemente a un conflicto militar. Si miramos el recurso estratégico, lo antedicho no es necesariamente promovido por quienes detentan el recurso sino por quienes lo demandan. Pero al mismo tiempo, esta perspectiva sirve para validar la idea de la defensa de los recursos naturales, aun cuando esos recursos que se dicen defender son parte de una estructura económica transnacional y son legalmente explotados por aquellos que adquirieron los derechos del estado. Además, si le sumamos la perspectiva de quien lo demanda, la defensa puede implicar pensar en el desarrollo de un instrumento militar que sea útil para defenderse de grandes potencias.

Sin embargo, al mismo tiempo limita los alcances del sesgo estrictamente economicista que pone énfasis en el comercio de materias primas y descarta otras acciones en el sistema internacional. También, lo hace en aquellas vinculadas a las distorsiones que pueden existir en este comercio y en las consideraciones temporales como la oportunidad de hacerse de recursos por la vía de la violencia o influyendo en un escenario político determinado que haga primar ciertos intereses por sobre otros. Para cerrar la idea, quienes detentan este tipo de posición presentan un espectro amplio de escuelas de pensamiento de las Relaciones Internacionales con extremos como: Bruce Russet y Harry Starr (1992), Jeffrey Sachs (2008) o Stephen Krasner (2002).

\section{Hidropolítica: el agua como recurso natural estratégico}

La dinámica que se impone cuando se analizan los recursos ha quedado previamente establecida en los puntos anteriores. Cuando pensamos en un recurso en particular, como puede ser el agua, todas las consideraciones se cristalizan en la palabra estratégico. A partir de las múltiples consideraciones existentes frente al recurso hídrico como fuente de vida, divisora de naciones, protectora de imperios, generadora de recursos económicos; diversos actores internacionales realizan consideraciones y establecen con quienes compite para asegurarse la provisión y el acceso al mismo. 
Si miramos la función seguridad, que se desprende del agua, podemos señalar que, en términos estructurales, en su función recurso de poder, que señala Morgenthau (1988, Pp. 103-107), la carencia de acceso o de la capacidad de provisión del mismo, por ejemplo, para la industria de alimentos, hace que el recurso sea mirado en clave de suma cero y que sea el sustrato de aquello que conocemos como dilema de seguridad. Por otra parte, para Mearsheimer (200 I, pp. 58-62), la distancia existente entre las grandes potencias por extensos bloques de agua sumado a la incapacidad de medios para poder revertir esa realidad da a los océanos la capacidad de barrera (stopping power of water) lo cual contribuye a la seguridad de quién posee acceso y a la inseguridad de quién no.

Estrategias como el anti acceso y la denegación de área se desarrollan en función de una creciente zona marítima conocida como "tierra de nadie" (Krepinevich et al., 2003), que recuerda a las zonas que quedaban entre las trincheras y que eran el espacio de encarnizados combates en la Primera Guerra Mundial. El agua es el escenario donde estrategias de acceso chocan con las de anti acceso, restándole a esa idea de supercarretera que brinda el comercio, la fuerza de integración con la que siempre el agua acompañó tanto ideas liberales como mercantilistas, al punto de gravitar en el análisis de la teoría política como lo revela Carl Schmitt (2007) en su libro Tierra y mar.

Además, el cambio climático también juega su rol en las percepciones acerca de la disponibilidad futura de los recursos acuíferos. Por ejemplo, los cambios relativos de temperatura afectan a los deshielos de alta montaña, aumentando el caudal y el curso de los ríos. Esta situación provoca alteraciones en las economías y en las vidas de quienes se encuentran en sus márgenes, generando a su vez, tensiones tanto en el interior de un país (si los cambios afectan a distintas localidades) o de manera internacional, si estos terminan afectando delimitaciones o cambios en la cantidad de recurso disponible si es compartido, lo cual, obliga a redimensionar la agenda entre quienes se enfrentan a este tipo de problemas.

El agua, en especial la dulce, se ha transformado en un recurso acumulativo de poder, de ahí que en los grandes espacios de hielos, tanto en el Ártico como en la Antártida, exista una mirada acerca del empleo de los cascos polares como fuente de abastecimiento de mega urbes que tienen cada vez mayores problemas para aprovisionarse de agua, donde los tratamientos de desalinización resultan prohibitivos en materia de costos. En este sentido, resulta importante remarcar que mientras que, en relación al agua salada, el acceso suele ser más importante que el control, en el caso del agua dulce, el control resulta central frente al acceso. Es por ello que, en relación al agua dulce cuando es compartida, se trata de generar mecanismos que den margen a la cooperación considerando que la pulsión primaria es la de competencia.

En definitiva, en la dimensión económica, tanto el agua dulce como la salada, tienen un valor intrínseco a las actividades humanas. Tanto como fuente de extracción de recursos, como en la acuicultura o la pesca abierta en alta mar, como para su uso en la agricultura y la crianza de ganado, existen múltiples valores económicos a ella asociados. Esto da lugar a la llamada hidropolítica la cual se define en términos de geopolítica alrededor del agua, sus usos y actores cercanos a ella. En este sentido, el mayor problema es el incremento de su escasez en varias partes del mundo en desarrollo, aunque la mayor dificultad radica en la distribución desigual de la misma, afectando el 
desarrollo de las economías locales y regionales.

La superposición de intereses también influye en las problemáticas de la economía política actual como consecuencia de la necesidad de grandes cantidades de agua para la industria, para la minería y para la obtención de recursos energéticos mediante técnicas de fractura (fracking). Las empresas que se dedican a la obtención de recursos energéticos mediante la técnica antes mencionada, revalorizan monetariamente diversos cursos de agua disparando tensiones que se presentan como opuestos pero que en los hechos demandan una mejor coordinación y generan discusiones sobre corrupción política y corporativa entre las comunidades locales y nacionales junto a los actores transnacionales.

Esta situación obliga a contextualizar la sustentabilidad, entendiéndola como la capacidad de generar un espacio común de gobernanza que evite el escalamiento de escaramuzas que suelen darse en relación al agua dulce, y que puede terminar en un conflicto armado abierto. Según The Pacific Institute, existen más de doscientos cursos de agua compartidos por dos o más estados, y al momento los conflictos entorno al agua suman un total de novecientos veintiséis, es un hecho que existen menos guerras por el agua si comparamos con otros recursos como el petróleo. De esos doscientos cursos de agua, cincuenta y siete se encuentran en África, treinta y cinco en América del Norte y un número similar en América del Sur, y cuarenta y ocho en Asia, cubriendo cerca del cincuenta por ciento del total de la masa territorial planetaria. Ese valor económico y de vida que tiene para todos los involucrados ha llevado a que existan cerca de trescientos tratados acerca de cómo lidiar con los problemas que el uso compartido de un recurso genera.

El agua no solo tiene un valor económico en sí mismo. Por ello, hay que considerar todas las industrias que se construyen alrededor del agua y que sirven para expandir el desarrollo económico y la libertad de movimiento. La industria naval, la alimenticia, los dragados y el turismo cerca de cursos de agua, cobran vida y sentido en función de aquello que invierten para lograr extraer los beneficios que esos cursos generan. Alrededor del agua, tanto dulce como salada, se ha generado el concepto de economía azul, el cual supone mirar a ambas en términos de espacios interrelacionados e interdependientes que se ven afectados y que afectan a su vez a la actividad humana. Existen también varias preguntas ligadas a la trasferencia de tecnología y de costos para tener una mayor disponibilidad de plantas de desalinización que permitan emplear el agua que cubre el setenta y cinco por ciento del planeta.

Las discusiones que tienen lugar en la presente etapa son las siguientes: ipodremos mantener un esquema funcional de cooperación en un mundo con recursos menguantes, en especial en lo que se refiere a los hábitats marítimos involucrados? ¿Se alterará fundamentalmente el balance entre la relación cooperación, conflicto y curso de aguas compartidas, generándose tensiones por la captura o desvío de cursos de agua de manera tal de evitar determinados efectos en una región en perjuicio de otra? ¿Se acelerará la captura de valor en materia de agua dulce proveniente de los deshielos en los cascos polares? Producto de ese aceleramiento, ise irán desvaneciendo los acuerdos previamente establecidos?

En este punto solo podemos dar respuestas parciales en relación a los interrogantes planteados. Si consideramos el primero el reporte de la United Nations Economic Commision for 
Europe (20I8), continúa remarcando los beneficios de la cooperación entre países que presentan cursos de agua compartidos a partir de la creciente capacidad de articulación de intereses sobre guiados por la comunidad internacional. Siguiendo en esta línea de ideas existe creciente evidencia que demuestra una divergencia hacia la cooperación en el caso de los cursos de agua en el continente, versus una creciente disputa por recursos de agua dulce en zonas como el Ártico y la creciente distribución desigual en diversas regionales de agua dulce tal como señalan Koenigk \& Fuentes-Franco (2019), situación que parece promover políticas claramente diferenciadas entre ambos espacios geográficos. Si consideramos la totalidad del fenomeno recurso hídrico como estrátegico, la situación aparece como indeterminada, aunque en cierto sentido un tanto más pesimista, ya que aun cuando hay cooperación entre países con recursos acuíferos compartidos, el número de acuerdos no se ha incrementado sustancialmente (Dinar et al., 20l6).

\section{Conclusión: en la hidropolítica, la ubicación también importa}

A modo de conclusión, podemos reafirmar lo siguiente: existe de manera simultánea un retorno a un discurso que refleja una tendencia general al conflicto por los RNE de las cuales el agua no queda exenta. Sin embargo y como demuestra el informe de la UNECE, los esfuerzos por mantener la gobernanza de este recurso hace que la situación se mantenga en un equilibrio débil pero estable. Desde el punto de vista de la defensa resultará relevante conocer la localización de los recursos a defender, quiénes lo comparten, cómo se piensa el acceso al mismo y cómo es la vecindad entre quienes se lo disputan. A medida que pasa el tiempo más se repite el consabido dicho, ¿qué hay de nuevo, viejo? Spykman $(1938$, p. 3) en su obra seminal Geography and Foreign Policy:

\footnotetext{
"Existen numerosos factores que condicionan la política de los estados. Algunos son temporales y otros permanentes. Algunos son obvios mientras que otros permanecen ocultos. Ellos son el factor geográfico, la estructura económica de los países, la composición étnica, la forma de gobierno y las percepciones (prejuicios) de los ministerios de relaciones exteriores".
}

El conflicto por el agua es acerca de la geografía en tanto mapas de posesión y uso del recurso y de las consecuencias que ello genera sobre la política. Múltiples son las consideraciones que entonces debemos realizar a partir de la ubicación, ya que debemos tener en cuenta, por ejemplo, si el recurso es compartido (como pueden ser los cursos de agua), si los límites entre países están sobre un recurso natural que puede ser explotado o depredado por un país a costa del otro, si la explotación de un recurso daña el medio ambiente de otro por la proximidad del mismo o si se afecta la geografía de un país para poder realizar una explotación de un recurso compartido. Ubicados en determinados estados, que a su vez están dentro de regiones, vecindarios, de los que no hay escapatoria, las consideraciones acerca de las condiciones que enfrenta la explotación del recurso van desde la posibilidad de crear la infraestructura necesaria para usufructuarlo, a la posibilidad de tener un acceso a la vía marítima y la accesibilidad del transporte terrestre, entre otras. VICA aumenta el error en el cálculo político, los riesgos de las acciones y el esquema de recompensas. 
Como se puede apreciar en las páginas precedentes el agua dulce tiene un lugar especial en la política internacional que se puede apreciar en el aumento de las tensiones que sobre este recurso aparece y el incremento en simultáneo de una gobernanza bilateral o multilateral con asistencia de organizaciones especializadas, que evite que una crisis escale a niveles no deseados. La idea de captura de valor no pasaría por el control, sino por el acceso. Sin embargo quedan espacios de la geografía mundial donde se ejercen controles férreos, como lo es en los Altos del Golán, pero a costa de una deslegitimación de la acción por sus controladores.

Asimismo no podemos escindir la dinámica de los ríos de la de los océanos. Se conectan y afectan mutuamente. La tecnología, sino también la capacidad económica y la fortaleza industrial, juegan un rol central en la incorporación de esta clase de espacio, ya que se abren las posibilidades de explotación de los recursos existentes y que no se podían explotar (sea por carencias tecnológicas o por razones de costos), ampliando así el valor estratégico de los diversos cursos de agua.

Los acuerdos que se lograron se mantienen a base de una convicción no escrita que es la de preservación mutua del bien común hídrico por el grado de vulnerabilidad al que se exponen los contendientes frente a un uso tal que resulte en un detrimento de quienes lo comparten. Pese a ello, en el seno de los estados, surgen tensiones entre diversos actores de la sociedad civil y agencias gubernamentales sobre las responsabilidades pasadas frente a su uso imprudente, contaminación previa, almacenamiento o alteraciones de cursos de agua con el objeto de favorecer o perjudicar determinados entornos políticos o negocios económicos. La mayor fuente de tensión se halla en el plano doméstico y no en el internacional, aunque eso no signifique que el mismo pueda eventualmente escalar al plano internacional. Al menos por ahora la prudencia realista ordena la praxis política en la forma en la que lidiamos con este recurso natural.

\section{Referencias}

Bremer, S. (2000).Who Fights Whom, When Where and Why? En Vazquez, J. (Ed.), What do we Know about war. Rowan Littlefield Publishers.

Brooks, S. ( I 997). Dueling Realism. International Organization, 3 (5I), 445-477.

Buzan, B. (2004). The United States and the Great Powers:World Politics in the XXI Century. Polity Press.

Conca, K. (2005). Governing Water: Contentious Politics and Global Institution Building. The MIT Press.

Dinar, S., Katz, D., De Stefano, L. y Blankespoor, B. (2016). Climate change and water variability: do water treaties contribute to river basin resilience? (Report No.WPS7855). World Bank. Recuperado de: https://documents.worldbank. org/en/publication/documents-reports/documentdetail/20990 I476193940390/climate-change-and-watervariability-do-water-treaties-contribute-to-river-basin-resilience.

International Energy Agency (2019). World Energy Balance Overview, Statistics. Recuperado de: https://iea.blob.core. windows.net/assets/8bd626fl-a403-4b I4-964f-f8d0f6 le0677/World_Energy_Balances_2019_Overview.pdf.

Klare, M. (2003). Guerras por los Recursos Naturales: El futuro escenario del conflicto global. Ediciones Urano.

Krasner, S. (julio, 2002). Troubled Society, Outlaw State, and Gradations of Sovereignty. Trabajo presentado en la Conferencia sobre Estados Fallidos, Stanford University.

Krepinevich,A., Watts, B. y Work, R. (2003). Meeting the Anti-Acess and Area-Denial Challenge. Center for Strategic and Budgetary Assessments. Recuperado de: https://csbaonline.org/uploads/documents/2003.05.20-Anti-AccessArea-Denial-A2-AD.pdf.

Koenigk,T.y Fuentes-Franco, R. (2019). Sensitivity of the Artic freshwater content and transport to model resolution. Clim Dym, 53, I765-I78I. Recuperado de: https://doi.org/I0.I007/s00382-019-04735-y.

Koubi,V., Spilker, G., Böhmelt, T. y Bernauer,T. (2014). Do natural resources matter for interstate and intrastate armed conflict? Journal of peace research, 5 I (2), 227-243. Recuperado de: https://doi.org/ I 0. I I 77/00223433 I 3493455.

McKay, A. (Director) (20I5). La Gran Apuesta [Film]. Plan B entertainment, Regency Enterprises.

Mearsheimer, J. (200I). The Tragedy of the Great Power Politics.W.W. Norton \& Company. 
Morgenthau, H. (1986). Política entre las naciones: La Lucha por el Poder y la Paz. Grupo Editor Latinoamericano.

Poelhekke, S. y Van Der Ploeg, F. (2010). Do Natural Resources Attract FDI? Evidence from non-stationary sector level data. (Working Paper No. 266). Recuperado de: https://www.dnb.nl/binaries/266\%20-\%20Do\%20Natural\%20 Resources\%20Attract\%20FDI_tcm46-242555.pdf.

Rosencrance, R. (1986). The Rise ofTrading State: commerce and conquest in the modern world. Basic Books.

Russet, B. y Starr, H. (1992). World Politics:The Menu for Choice. Freeman and Company.

Sachs, J. (2008). Economía para un planeta abarrotado. Editorial Debate.

Schmitt, C. (2019). Tierra y Mar: una reflexión sobre la historia universal. Editorial Trotta.

Schweller, R. (1999). Realism and the Present Great Power System: Growth and Positional Conflict Over Scarce Resources. En Kapstein, E. y Mastanduno, M. (Eds.). Unipolar Politics: Realism and State Strategies after the Cold War. Columbia University Press.

Spykman, N. (1938). Geography and Foreign Policy I. The American Political Science Review, I (32), 28-50. Recuperado de: https://doi.org//0.2307//949029.

United Nations Economic Commission for Europe. (2018). Identifying, assessing and communicating the benefits of transboundary water cooperation (ECE/MP.WAT/NONE/I I). Recuperado de: https://www.unece.org/fileadmin/ DAM/env/water/publocations/WAT_NONE_I I_benefits/ECE.MP.WAT.NONE.I I_ENG:I826722_E_web. pdf.

Van Evera, S. (1999). Causes of War: Power and the Roots of Conflict. Cornell University Press.

Villalba, G. (2009). El atlas de Le Monde diplomatique III (Ist Ed.). Capital Intelectual. 


\section{RELACIONES INTERNACIONALES}

Revista académica cuatrimestral de publicación electrónica

Grupo de Estudios de Relaciones Internacionales (GERI)

Universidad Autónoma de Madrid, España

https://revistas.uam.es/relacionesinternacionales

ISSN 1699 - 3950

f facebook.com/RelacionesInternacionales

3. twitter.com/RRInternacional 